\title{
UNCLASSIFIED CASES OF TIBIAL HEMIMELIA; SHOULD WE MODIFY THE CLASSIFICATION SYSTEMS?
}

\author{
Ahmed Abdelaal $^{(*)}$, Wael Salama,Yasser Soror, Shazly Moussa \\ Orthopaedic dept, Faculty of Medicine, Sohag University, Sohag, Egypt \\ "E-mail: ahmedallam201@gmail.com
}

Received 15/1/2021

Accepted 22-/5/2021

\begin{abstract}
Tibial hemimelia is a rare congenital anomaly, may be in isolated form or combined with other congenital anomalies. Different classification systems had been used for classification. While inferior tibio-fibular joint diastasis is a well-known accompanying deformity in subtypes of different classification systems; complete diastasis of tibia and fibula is not included in either of classification systems. Case 1: A 15 days infant presented to our unit with complete diastasis of tibia and fibula from the level of the knee joint. Case 2: A four weeks old boy with a partial distal diastasis of tibia and fibula from the level of mid leg downward, with almost completely separate skin envelop for each part of the bone. A search in the English literature for reported cases with complete diastasis of tibia and fibula and different classification systems for tibial hemimelia was done. No reported cases in the literature with complete diastasis of tibia and fibula finally, we suggest modifying the current classification systems for tibial hemimelia to include complete diastasis of tibia and fibula as a variant.
\end{abstract}

Keyword: Tibial hemimelia, Tibiofibular, Diastasis

\section{Introduction}

Congenital longitudinal deficiency of the tibia is very rare disease with an incidence of 1 in 1,000,000 live births [1,2]. It was first named Tibial Hemimelia by Billroth in 1861 [3,4]. Tibial hemimelia is characterized by partial or complete absence of the bone. It usually occurs as a solitary anomaly but may be part of a complex syndrome [5]. Different classification systems had been used for classification of tibial hemimelia. Jones, Barnes and
Lloyd- Roberts [6] in 1978 described a four types classification system for tibial hemimelia, in which the tibia may be totally absent, distal part is absent, proximal part is absent or distal part is absent with distal tibio-fibular diastasis as type I, II, III or IV respectively. Kalamchi and Dawe [7] in 1985 classified tibial hemimelia into three groups: totally absent, partially absent, or dysplastic with diastasis of the distal tibio-fibular joint. In 2008, Weber 
[8] believed that these earlier classifications were no longer useful for present-day requirements and suggested a new classification reflecting the severity of the condition morphology, clinical prognosis and the importance of any cartilaginous anlage. In either of the former classification systems, diastasis of the inferior tibio-fibular joint appears to be related to different types of tibial hemimelia $[9,10]$. However, congenital isolated Congenital diastasis of the inferior tibio-fibular joint also presents as a clinical problem such as talipes equinovarus, ankle diastasis, and leg-length inequality as many surgeons agree [11], despite the exact etiology is not well defined. We report two cases of tibial hemimelia with complete diastasis of the tibia and fibula with separate skin envelop for each bone, the first case has complete diastasis, while the second case has partial diastasis, where the deformity could not be classified by any of the above systems and hadn't been reported before.

\section{Case Report}

\subsection{Case one}

A fifteen days old boy was presented to our pediatric orthopedic surgery unit with a deformed and bifid left leg. His parents were non-consanguineous and apparently healthy and his only brother was normal. His prenatal history was irrelevant. The baby looks generally well. The left knee was sub-laxed with complete diastasis of tibia and fibula from the level of knee joint downward, with almost completely separate skin envelop for each bone, the fibula attain a flexed abducted position, while the tibia attain a flexion adduction position. Foot was articulated via dislocated joint with the fibula, while the tibia has no articulation with foot and widely separated from the fibula. Foot is supinated 180 degree with deficient first ray and the foot was smaller than the right side but most of the bones of the foot were present. The dorsalis pedis and tibialis posterior pulses were absent, but striking the foot seems to elicit normal reflexes. There was partial active plantar flexion of the toes but no dorsiflexion. There was some movement in the subtalar or midfoot joints and passive pronation of the foot was possible. Patella could be palpated, a web is presented between the both bones of the leg. No hip subluxation or dislocation could be detected using the screening Barlow and Ortolani tests. Examination of the upper limbs and spine showed no abnormality, Routine abdominal and chest examination by a pediatrician was done and revealed no abnormality. Hip ultrasonographic examination was performed and showed no dislocation or subluxation in either hip joint. Abdominal ultrasonography as well as chest echocardiography was done and revealed no abnormality. Clinical appearance of the limb is shown in fig. (1). Radiographs and computed axial tomography showed a proximally displaced fibula with diastasis of both the proximal and distal tibio-fibular joints, widely separated tibia and fibula, ankle joint is not present, deficient medial ray of the foot and medial femoral condyle hypoplasia, fig. (2). 

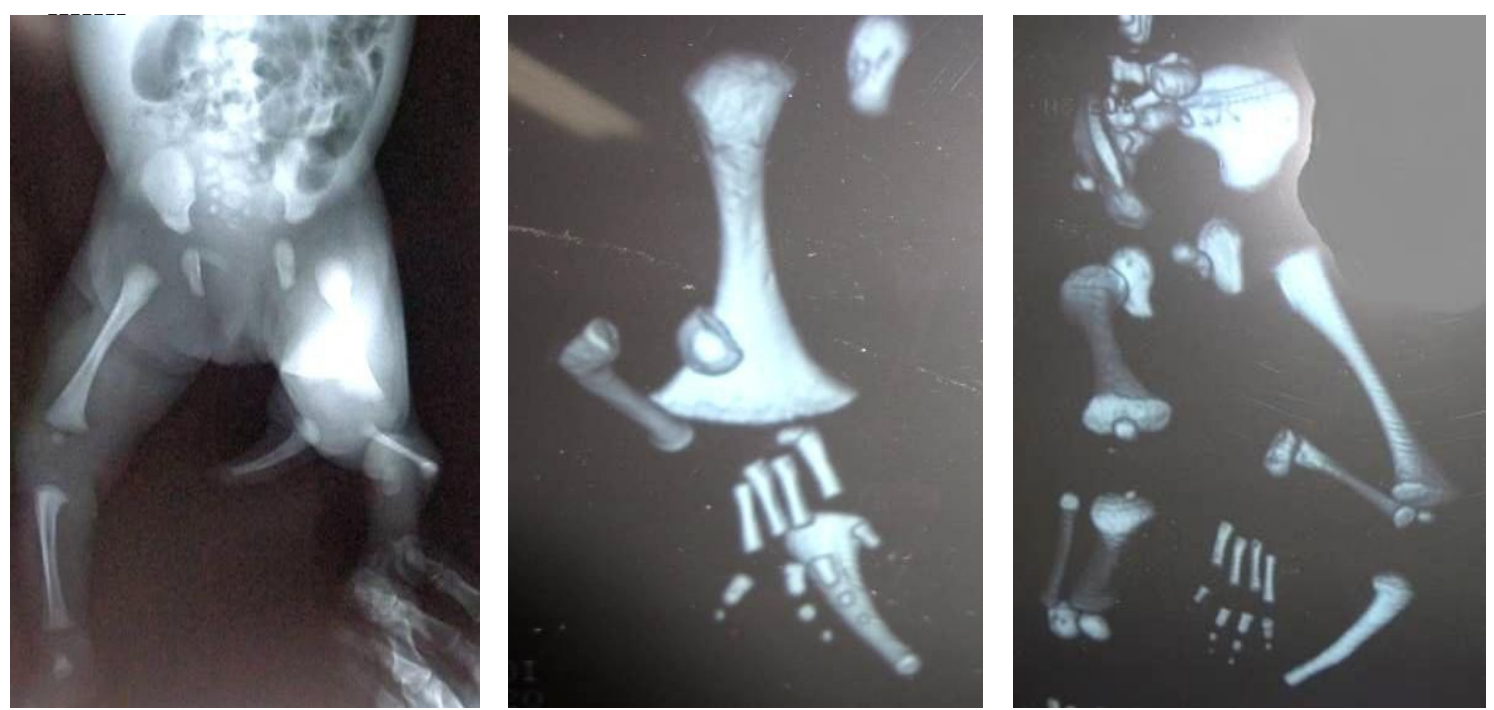

Figure (1) shows radiograph and CT scan of case 1; the complete diastasis of tibia and fibula
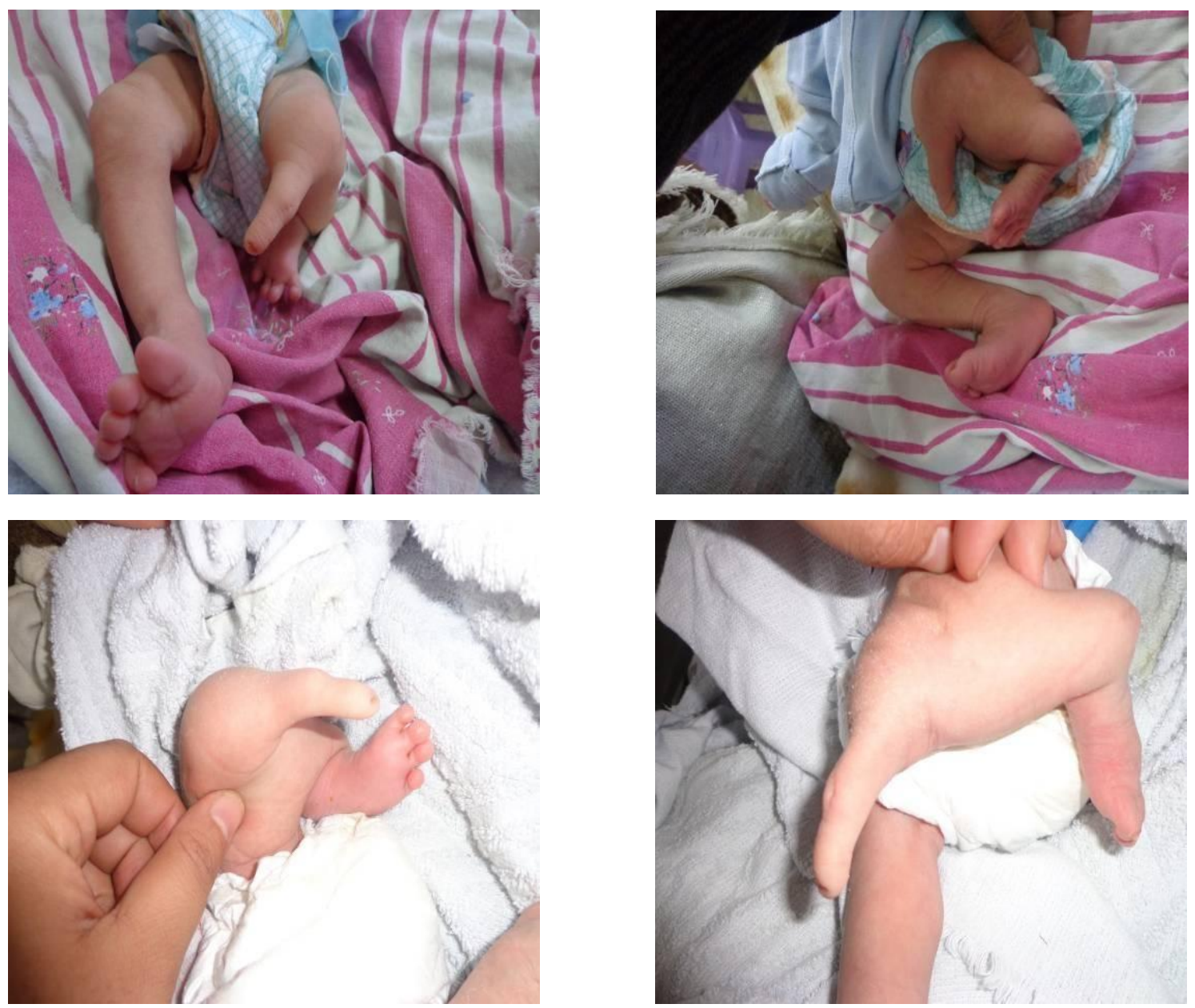

Figure (2) shows clinical photos of case 1; the complete diastasis of tibia and fibula

\subsection{Case two}

A four weeks old boy with a deformed right leg. There was a distal diastasis of tibia and fibula from the level of mid leg downward, with almost completely separate skin envelop for each part of the bone, the fibula attain a slightly flexed abducted position, articulating with the smaller sized inverted foot. Foot was articulated through 
a hyper-flexible joint with the rudimentary fibula, while the tibia hasno articulation with foot and widely separated from the fibula. No other apparent congenital anomalies, fig. (3). Treatment possibilities include amputation of the tibial stump with
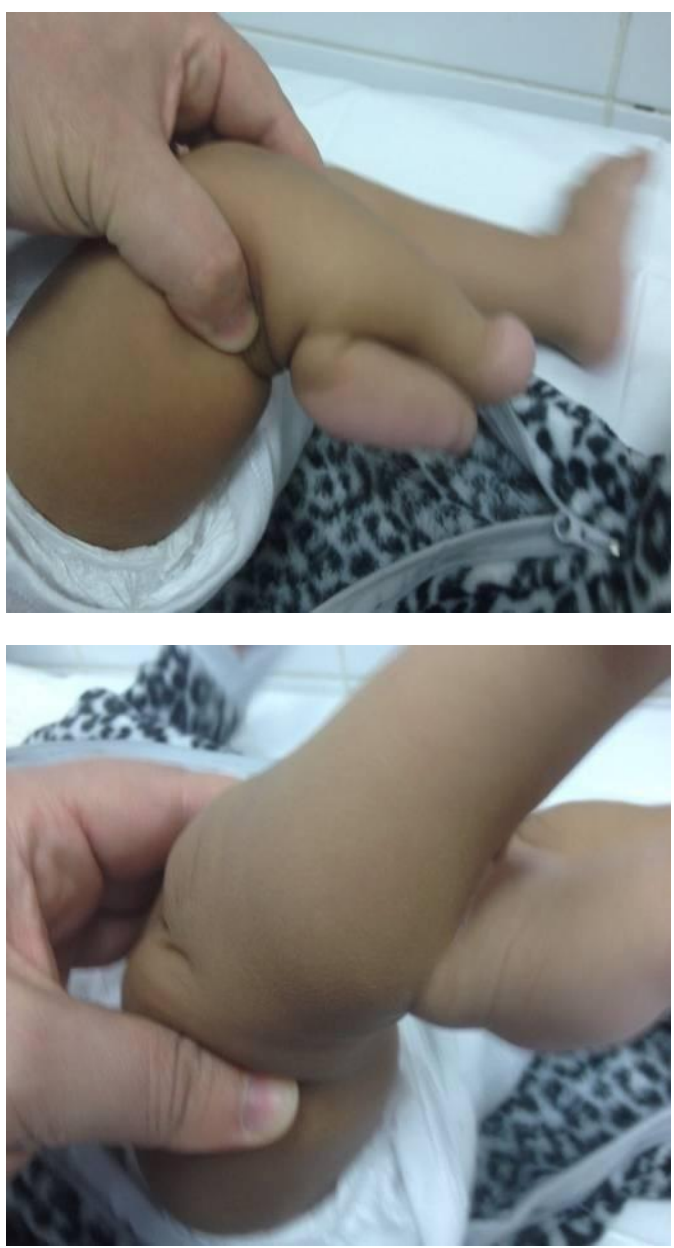

tibialization of fibula. However disarticulation knee with prosthesis replacement is not a far possibility at the suitable time for either option. Lack of literature guidelines to treat such cases makes the decision making a real clinical dilemma.
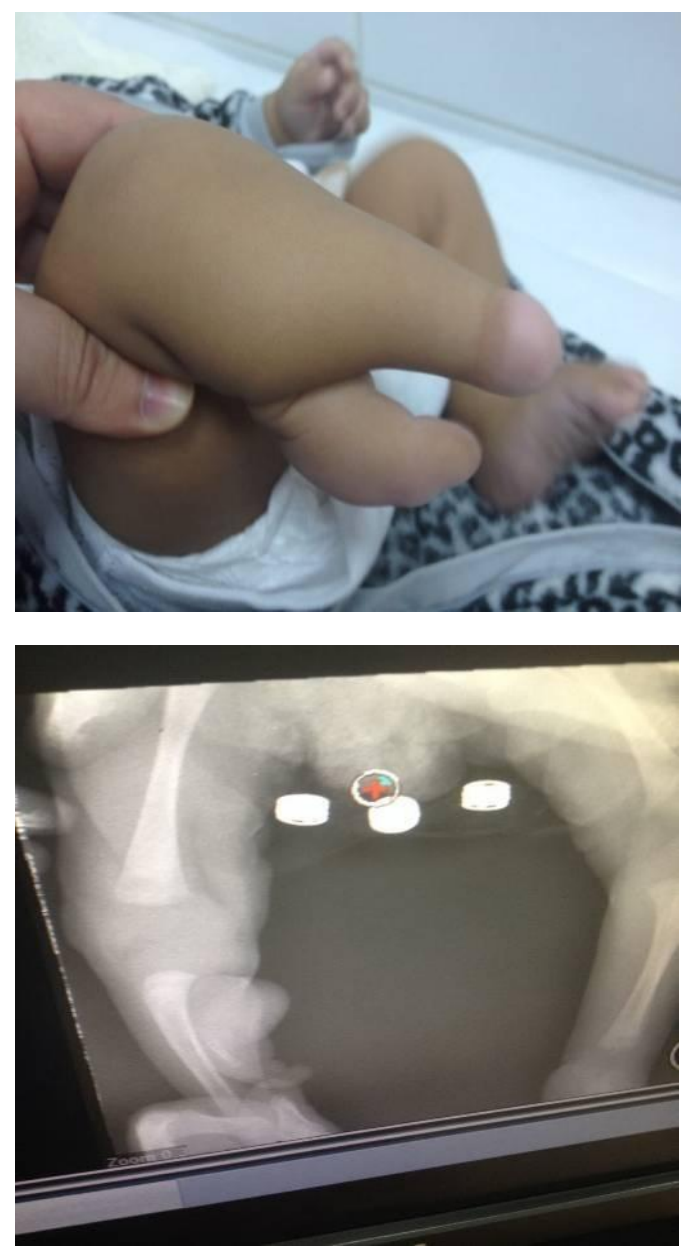

Figure (3) shows clinical and radiographic photo of case 2; the partial diastasis of tibia and fibula

\section{Discussion}

Different classification systems for tibial hemimelia is present Although there is no many articles published about the gene location for tibial hemimelia $[12,13]$. Distal tibio-fibular diastasis is a component of subtype in more than one of these classification systems. But as our case has complete diastasis, not only distal, and separate soft-tissue envelop for both tibia and fibula, our case does not fulfill the criteria of any recognized classification system. However, up to date no classifica- tion system entails the complete diastasis of tibia and fibula as a variant. Jones type 4 , in which there is distal tibio-fibular diastasis and foot is usually positioned between the divergent tibia and the fibula seems to be a possible classification. Our opinion to add these cases description to Kalamchi and Dawe classification as Type IV, As they mentioned different degrees of tibio-fibular diastasis within the same soft tissue covering in type III. 


\section{References}

[1]Brown, F., (1971). The brown operation for total hemimelia tibia. In: Aitken, G. (ed.) Selected lower-limb anomalies. National Academy of Sciences, Washington, pp. 20-28.

[2]Weber, M., Schroder, S., Berdel, P., et al. (2005). Register zur bundesweiten Erfassung angeborener Gliedmaßenfehlbildungen. Z Orthop. 143: 1-5.

[3] Billroth, T., (1861). Ueber einige durch Knochendefecte bedingte Krummungen des Fusses. Arch Klin Chir. 1: 252-268

[4] Dankmeijer, J., (1935). Congenital absence of the tibia. Anat Rec. 62: 179-194

[5] Mirzatolooe, F. (2011). Tibial hemimelia with separate soft-tissue cover of the tibia and fibula. J. Bone Joint Surg Br. 93 (7): 990-1000.

[6] Jones, D., Barnes, J., Lloyd-Roberts, G. (1978). Congenital aplasia and dysplasia of the tibia with intact fibula: Classification and management. J. Bone Joint Surg Br. 60-B: 31-39.

[7] Kalamchi, A., Dawe, R., (1985). Congenital deficiency of the tibia. $\boldsymbol{J}$. Bone Joint Surg Br. 67 (4): 581-584.
[8] Weber, M., (2008). New classification and score for tibial hemimelia. J. Child Orthop. 2: 169-175.

[9]Bose, K., (1976). Congenital diastasis of the inferior tibio-fibular joint. J.Bone Joint Surg Am., 58, pp. 886-887.

[10] Fernandez-Palazzi, F., Bendahan, J., Rivas, S., (1998). Congenital deficiency of the tibia: A report on 22 cases. $\boldsymbol{J}$. Pediatr Orthop. 7: 298- 302.

[11] Tuli, S., Varma, B., (1972). Congenital diastasis of the tibio-fibular mortise. J. Bone Joint Surg Br. 54, 346- 350.

[12] Kantaputra, P., Chalidapong, P., (2000). Are triphalangeal thumb-polysyndactyly syndrome (TPTPS) and tibial hemimeliapolysyndactyly-triphalangeal thumb syndrome (THPTTS) identical?: A father with TPTPS and his daughter with THPTTS in a Thai family. Am J. Med Genet. 93: 126-131.

[13] Stevens, C., Moore, C., (1999). Tibial hemimelia in Langer-Giedion syndrome: Possible gene location for tibial hemimelia at 8q. Am J. Med Genet. 85: 409-412. 